\title{
Dual-color, break-apart fluorescence in situ hybridization for EWS gene rearrangement distinguishes clear cell sarcoma of soft tissue from malignant melanoma
}

\author{
Rajiv M Patel ${ }^{1}$, Erinn Downs-Kelly ${ }^{2}$, Sharon W Weiss ${ }^{1}$, Andrew L Folpe ${ }^{1}$, \\ Raymond R Tubbs $^{2}$, Ralph J Tuthill ${ }^{2}$, John R Goldblum ${ }^{2}$ and Marek Skacel ${ }^{2}$ \\ ${ }^{1}$ Department of Pathology and Laboratory Medicine, Emory University, Atlanta, GA, USA and ${ }^{2}$ Division \\ of Pathology and Laboratory Medicine, The Cleveland Clinic Foundation, Cleveland, OH, USA
}

\begin{abstract}
Clear cell sarcoma of soft tissue (malignant melanoma of soft parts) is a soft tissue sarcoma with melanocytic differentiation that typically occurs in the tendons and aponeuroses of young adults. As demonstrated by cytogenetics and reverse-transcriptase polymerase chain reaction, between $70 \%$ and over $90 \%$ of clear cell sarcomas have a t(12;22) translocation, fusing the EWS and ATF1 genes on chromosomes 22q12 and 12q13, respectively. Identification of this translocation distinguishes clear cell sarcoma from histologic mimics, most importantly conventional malignant melanoma. We report our experience with a commercially available, dualcolor, break-apart fluorescence in situ hybridization (FISH) probe, which allows detection of EWS (22q12) gene rearrangement in formalin-fixed, paraffin-embedded tissues. Histologically and immunophenotypically wellcharacterized cases of clear cell sarcoma $(n=10)$ and malignant melanoma $(n=32)$ were evaluated with a 22q12 dual-color, break-apart probe (Vysis, Downer's Grove, IL, USA), which spans the known common breakpoints in the EWS gene on chromosome 22 (introns 7-10). Signals from tumor cell nuclei were counted under a fluorescence microscope and the presence of red-green break-apart signals was recorded. Of the clear cell sarcoma cases, seven of 10 showed evidence of an EWS gene rearrangement with a mean of $81.6 \%$ positive cells per sample (range: $60-95 \%)$. All cases of malignant melanoma $(n=32)$ showed virtually absent break-apart signals in the EWS gene (less than $4 \%$ cells per case). FISH detects EWS gene rearrangement in a substantial proportion of clear cell sarcomas, with excellent specificity. Importantly, EWS FISH is negative in malignant melanoma, a clinically dissimilar tumor, which may closely mimic clear cell sarcoma histologically and immunohistochemically. As the studied probe can be utilized in routinely processed tissue, FISH provides an excellent alternative to reverse-transcriptase polymerase chain reaction in cases where fresh tissue is unavailable.
\end{abstract}

Modern Pathology (2005) 18, 1585-1590. doi:10.1038/modpathol.3800503; published online 7 October 2005

Keywords: fluorescence in situ hybridization; clear cell sarcoma of soft tissue; malignant melanoma of soft parts; malignant melanoma

Clear cell sarcoma of soft tissue (malignant melanoma of soft parts) is a rare soft tissue sarcoma with melanocytic differentiation first described by Enzinger ${ }^{1}$ in 1965 . The tumor originates predominantly in the tendons, aponeuroses, and fascial

Correspondence: Dr RM Patel, MD, H-185A Department of Pathology, Emory University Hospital, 1364 Clifton Rd NE, Atlanta, GA 30322, USA.

E-mail: rmpate5@emory.edu

Presented in part at the 94th Annual Meeting of the United States and Canadian Academy of Pathology, San Antonio, TX, USA, Tuesday, March 1, 2005.

Received 26 May 2005; revised and accepted 1 September 2005; published online 7 October 2005 structures of the extremities of young adults. ${ }^{1-5}$ Clear cell sarcoma shares morphologic, immunohistochemical, ultrastructural, and molecular features with malignant melanoma. ${ }^{2,6-10}$

In contrast to malignant melanoma, a unique and recurrent $\mathrm{t}(12 ; 22)(\mathrm{q} 13 ; \mathrm{q} 12)$ involving the $E W S$ gene has been identified in $70 \%$ to over $90 \%$ of clear cell sarcomas by cytogenetics, reverse-transcriptase polymerase chain reaction (RT-PCR) looking for the resultant EWS-ATF1 fusion protein, and by fluorescence in situ hybridization (FISH). ${ }^{11-19}$ Clear cell sarcoma is rare and the true incidence of this neoplasm is not well established; however, the increased utilization of molecular studies for the 
identification of the characteristic translocation may help to better define its incidence in the future. Although clear cell sarcoma is rare relative to malignant melanoma, it should be a diagnostic consideration in patients who present with a tumor with the requisite histologic features and no history of a primary cutaneous or mucosal melanoma. The significance of distinguishing between these two entities is of great consequence in light of the fact that metastatic melanoma (AJCC stage IV melanoma) has a median survival rate of 7.5 months and an estimated 5-year survival rate of $6 \%,{ }^{20}$ a prognosis much different from clear cell sarcoma, which in the few studies with long-term follow-up and large cohorts, has 5, 10, and 20 year survival rates of $67 \%, 33 \%$, and $10 \%$, respectively. ${ }^{21}$

A dual-color, break-apart FISH probe, which allows detection of EWS (22q12) gene rearrangement in formalin-fixed, paraffin-embedded tissues, has recently become commercially available and could potentially serve as an alternative ancillary diagnostic tool in situations where fresh tissue is not available for classical cytogenetics and/or RT-PCR. Herein we report our experience utilizing this probe for distinguishing clear cell sarcoma from its most important histological mimic, malignant melanoma.

\section{Materials and methods}

The study was performed on histologically and immunophenotypically well-characterized cases of clear cell sarcoma $(n=10)$ and malignant melanoma $(n=32)$, using both conventional whole-tissue sections and a tissue microarray. After Institutional Review Board approval, tissues were obtained from resection specimens or excisional biopsies from the Department of Anatomic Pathology at the Cleveland Clinic Foundation and the soft tissue consultation service of the Department of Pathology at Emory University. All cases were reviewed by three experienced soft tissue pathologists (SWW, JRG, and ALF) and an experienced dermatopathologist (RJT).

All tissue was neutral buffered formalin-fixed and paraffin-embedded. Whole-tissue sections stained with hematoxylin and eosin were evaluated for tumor and appropriate areas were scored for tissue microarray construction. Cases were arrayed in duplicate $1.5-\mathrm{mm}$ cores. After array construction, $4-\mu \mathrm{m}$ sections were placed on electrostatically charged slides (Superfrost; Fisher; Hampton, NH, USA), with the last section stained with hematoxylin and eosin to confirm the presence of tumor. The clear cell sarcoma whole-tissue sections and the tissue microarray were then subjected to direct FISH using the EWS (22q12) dual-color, break-apart probe (Vysis, Downer's Grove, IL, USA), which spans the known common breakpoints in the EWS gene on chromosome 22 (introns $7-10$ ). The $1100 \mathrm{~kb}$ probe specific for the $3^{\prime}$ (telomeric) side of EWS was labeled in Spectrum Green and the $500 \mathrm{~kb}$ probe specific for the $5^{\prime}$ (centromeric) side of $E W S$ was labeled in Spectrum Orange. FISH was performed according to the manufacturer's instructions and established laboratory protocol. The tissue microarray was then scored by evaluating 100 tumor cell nuclei per case with fluorescence microscopy. Cells that have the chromosomal rearrangement will have a discreet red signal distanced from a discreet green signal, indicating a translocation involving one EWS allele, while the second allele will be intact with a red and a green signal which are opposed (less commonly, both chromosomes in the same cell may show this rearrangement and occasionally, more than two copies of chromosome 22 may be seen with a variable number of rearranged chromosomes present). The interpretation of intact and split signals was based on generally accepted guidelines recommended by Vysis and used for all other commercially available break-apart FISH assays in clinical laboratories performing testing using this method. This approach requires the space between two signals to be greater than one signal width in order to be considered a split signal. Cells without the rearrangement will have two sets of red and green opposed signals, indicating two intact EWS alleles. In keeping with other FISH assays validated for paraffin-embedded tissue in our laboratory, a positive result was reported when greater than $10 \%$ of the tumor nuclei had evidence of an EWS rearrangement. To avoid false-positives resulting from nuclear truncation occurring in a subset of cells in paraffin-embedded samples, only tumor nuclei with all four signals present were evaluated, and overlapping cells indistinguishable as separate nuclei were excluded from the analysis.

\section{Results}

Our laboratory has validated the EWS (22q12) probe from Vysis, Inc. in formalin-fixed and paraffinembedded tissues. Testing for the presence of an EWS-FLI1 fusion transcript by RT-PCR was undertaken in fresh-frozen tissues from cases indexed as Ewing's sarcoma $(n=8)$, alveolar rhabdomyosarcoma $(n=2)$, lymphoblastic lymphoma $(n=1)$, and small cell carcinoma $(n=1)$. The EWS-FLI1 fusion transcript was found to be present in $8 / 8(100 \%)$ of the Ewing's sarcoma cases, while the fusion transcript was not identified in the other neoplasms studied. FISH using the EWS (22q12, Vysis, Inc.) break-apart probe was then performed in the corresponding formalin-fixed, paraffin-embedded tissues from these cases. A total of 100 tumor nuclei per case were evaluated, blinded to the RT-PCR results. All eight cases of Ewing's sarcoma had evidence of a rearrangement involving $E W S$, with a 
mean of $70 \%$ positive nuclei per 100 tumor nuclei (range: $62-85 \%$ positive nuclei per 100 tumor nuclei, mean: 70.25, s.d.: 8.76). The remaining neoplasms were essentially devoid of any rearrangement involving the EWS gene (range: $1-4 \%$ positive nuclei per 100 tumor nuclei, mean: 1.8, s.d.: 1.48). This distribution establishes a probe-specific normal range of less than or equal to $4 \%$ (4.76 respectively, if two standard deviations from the mean are used for calculation of this cutoff), and establishes that the EWS (22q12) probe shows excellent sensitivity and specificity for identifying the common breakpoints at 22q12. ${ }^{22}$

All 10 cases of clear cell sarcoma as well as the 32 cases of malignant melanoma were successfully analyzed. The tissue microarray had duplicate cores from five clear cell sarcoma and 32 malignant melanoma cases; all cores were present for evaluation. The concordance between the duplicate cores was excellent, with the clear cell sarcoma cases having a Pearson coefficient of 0.99 and the malignant melanoma cases having a Pearson coefficient of 0.97 .

None of the patients with clear cell sarcoma had a previous history of malignant melanoma. The characteristic morphology of clear cell sarcoma consisted of nests or short fascicles of rounded or fusiform cells with clear cytoplasm separated by delicate fibrous $\operatorname{septae}^{23}$ (Figure 1a-C). All 10 cases of clear cell sarcoma were immunoreactive for S-100 and melanocyte-specific marker HMB-45, with three cases focally reactive for Melan-A (Figures 2 and 3). Although the morphology varied, malignant melanoma cases were characterized by an epidermal junctional component and immunoreactivity for S-100 and at least one melanocyte-specific marker.

Of the clear cell sarcomas, 7/10 cases showed evidence of an EWS gene rearrangement with a mean of $81.6 \%$ positive cells per sample (range: 60-95\%) (Figure 4). RT-PCR correlation was available for two of the clear cell sarcomas (performed as a send-out test at the time of diagnosis to Dr Frederick Barr's Molecular Pathology Laboratory at the University of Pennsylvania, Philadelphia, PA, USA). The clear cell sarcoma FISH and RT-PCR results were correlative, with one EWS FISH-positive case having the EWS-ATF1 fusion transcript, and one EWS FISH-negative case not having the EWS-ATF1 fusion transcript identified. All 32 cases of malignant melanoma showed virtually absent break-apart signals in the EWS gene region (fewer than $4 \%$ cells per case) (Table 1).

Two of the clear cell sarcoma cases that were represented on the tissue microarray were negative for the EWS gene rearrangement by FISH. To ensure that sampling was not a cause for this result, whole tissue sections from these cases were reassessed with the EWS FISH probe and the results remained the same.
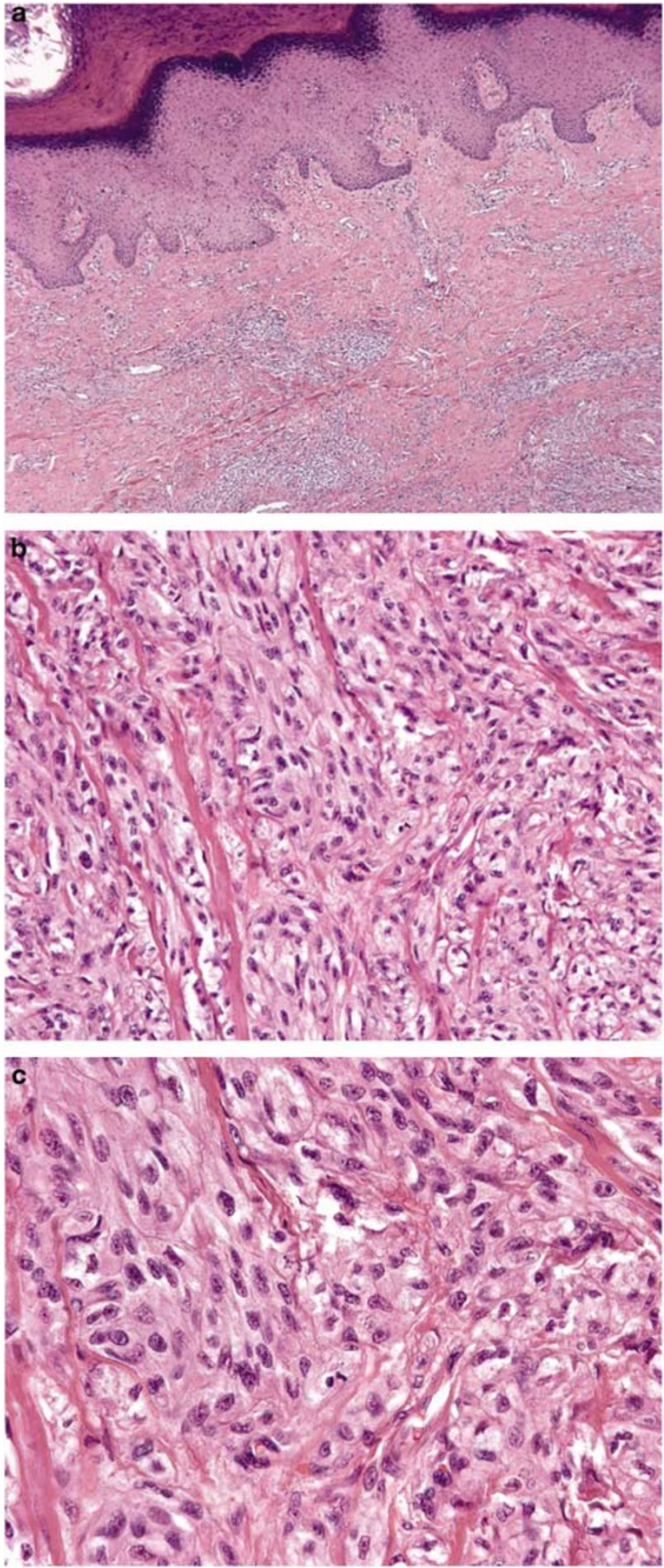

Figure 1 (a) Clear cell sarcoma of soft tissue identified by chromosomal rearrangement involving the EWS gene (22q12). Acral skin with normal epidermis and an ill-defined infiltrative process present in the reticular dermis are seen (magnification, $\times 20$ ). (b) Dermal infiltrate composed of spindled eosinophilic cells with haphazard arrangement and intersecting dense collagen bands is present (magnification, $\times 200$ ). (c) Dermal infiltrate consists of cells with round to oval nuclei and prominent nucleoli (magnification, $\times 400$ ). 


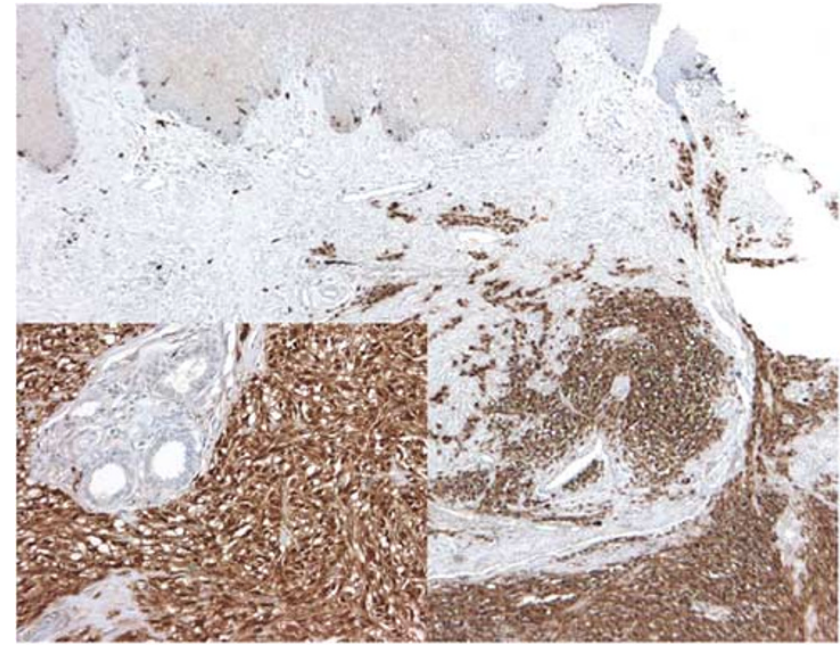

Figure 2 Clear cell sarcoma of soft tissue with intense S-100 immunoreactivity in the neoplastic cells in the reticular dermis, dendritic cells are highlighted in overlying dermis (magnification $\times 50$ ). Inset, S-100 staining both nuclear and cytoplasmic compartments of neoplastic cells (magnification $\times 200$ ).

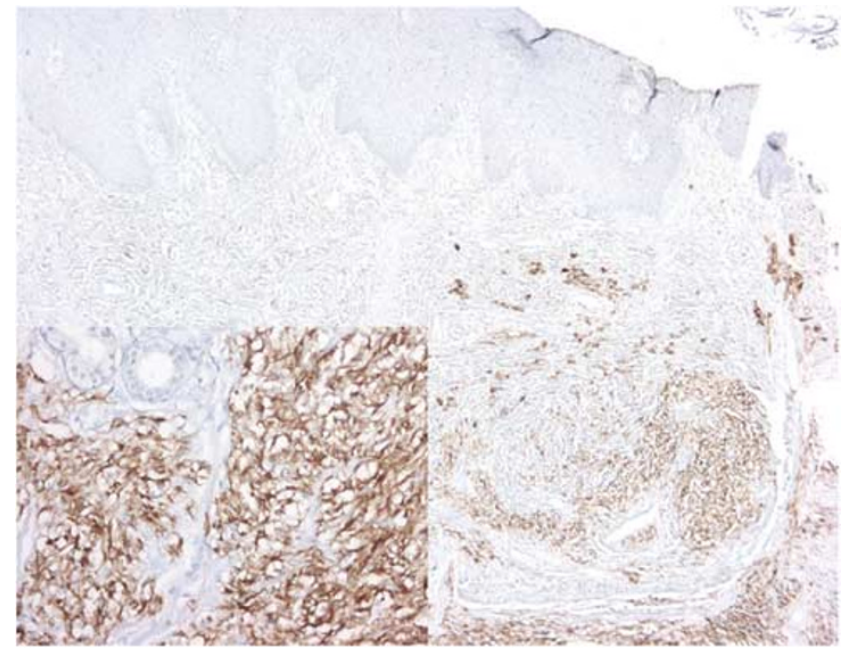

Figure 3 Clear cell sarcoma of soft tissue with HMB-45 immunoreactivity in neoplastic cells in the dermis (magnification $\times 50$ ). Inset, HMB-45 reactivity in the neoplastic cells showing a membranous staining pattern (magnification $\times 200$ ).

\section{Discussion}

Clear cell sarcoma of soft tissue and malignant melanoma share morphological, immunohistochemical, ultrastructural, and molecular features. Both can demonstrate melanin synthesis and are immunoreactive for S-100 protein, and melanocyte-specific markers HMB-45 and MITF, among others. ${ }^{6,9,19,24}$ Shared ultrastructural features of melanocytic differentiation include cytoplasmic melanosomes and the presence of an external lamina. ${ }^{6}$ At the molecular level, alterations of the p16INK4a/ p14ARF pathway are present in both clear cell sarcoma and malignant melanoma. ${ }^{25}$ Although originally reported to be genetically distinct from malignant melanoma, ${ }^{16}$ recent genomic profiling

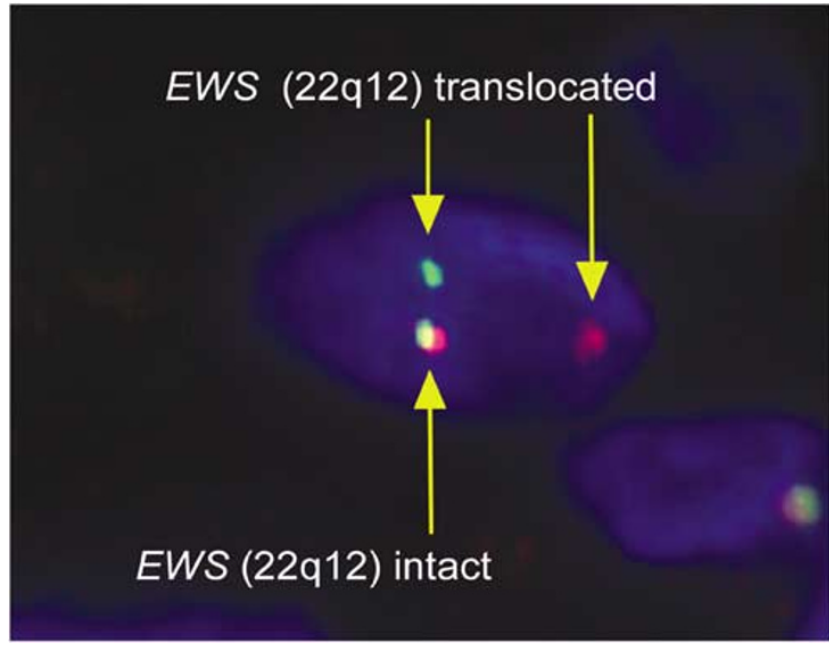

Figure 4 Dual-color, break-apart interphase FISH in clear cell sarcoma of soft tissue. Within a single nucleus, split red and green signals indicate the presence of an EWS (22q12) gene rearrangement involving one chromosome. The second signal is fused, indicating an intact 22q12 allele of the second chromosome in this cell (magnification $\times 1000$ ).

Table 1 EWS gene rearrangement by diagnosis

Diagnosis $\quad$ EWS (22q12) gene rearrangement

Clear cell sarcoma $\quad 7 / 10(70 \%)$ (range $60-95 \%+$ cells per case) Malignant melanoma $0 / 32$ (range $0-4 \%+$ cells per core)

data suggest that clear cell sarcoma is a subtype of melanoma. ${ }^{26}$ However, in contradistinction to malignant melanoma, clear cell sarcoma harbors EWS gene rearrangements in up to $90 \%$ of cases. ${ }^{11-19,27}$ Thus, translocations involving EWS are diagnostically useful for distinguishing clear cell sarcoma from its most important mimic malignant melanoma. ${ }^{19}$ Although not a hypothesis tested in this study, clear cell sarcoma could conceivably be distinguished from benign melanocytic proliferations that mimic malignant melanoma, such as cellular blue nevi, deep penetrating nevi, and proliferative nodules arising within congenital nevi, as these lesions have not been reported to contain EWS gene rearrangements. ${ }^{28,29}$

The $\mathrm{t}(12 ; 22)$ translocation fuses the EWS gene on chromosome 22 with the ATF gene on chromosome $12 .^{30}$ This translocation was first demonstrated by Bridge and co-workers ${ }^{11,31}$ by classical cytogenetics, and subsequently confirmed by others ${ }^{12-14}$ in shortterm and continuous cell culture lines. ${ }^{32,33}$ To date, four separate fusion transcripts have been described in the literature. ${ }^{17,18}$ The most common fusion transcript, found in approximately $87 \%$ of trans location-positive cases with fusion structure data, ${ }^{17}$ consists of an EWS exon 8 fusion to ATF1 codon 65, as described by Zucman et al. ${ }^{34}$ The other three EWS-ATF1 variant transcripts are rare, accounting for approximately $10 \%$ of cases. ${ }^{35}$ The EWS-ATF-1 
transcripts encode a constitutive transcriptional activator ${ }^{36}$ whose mechanism of action appears to be targeted repression of the CBP/p300 transcriptional coactivator, resulting in loss of function of p53. ${ }^{37}$ Although not diagnostically helpful, polysomy of chromosome 8 is another common cytogenetic abnormality in clear cell sarcoma. ${ }^{11,31}$ Other molecular characteristics which may have diagnostic utility in the future include activating mutations in the $B R A F$ gene, which have been demonstrated in a significant percentage of malignant melanoma, but not clear cell sarcoma, ${ }^{38}$ and the protein product of the epidermal growth factor receptor ERBB3 gene, which has been reported to be a marker of clear cell sarcoma. ${ }^{39}$

A diagnostic dilemma arises when the pathologist is confronted with a malignant melanoma of unknown primary, where histologic, immunophenotypic, and clinical features resemble clear cell sarcoma. In this circumstance, cytogenetic examination of fresh tissue remains the gold standard, as it may reveal prognostically relevant karyotpic changes in addition to the $\mathrm{t}(12 ; 22)(\mathrm{q} 13 ; \mathrm{q} 12) .{ }^{27}$ When fresh tissue is unavailable, FISH and/or RT-PCR are methods applicable to formalin-fixed, paraffinembedded tissues. Unfortunately, specific problems arise from RT-PCR of archival tissue due to degradation of RNA and insufficient extraction efficiency. ${ }^{40}$ For this reason, RT-PCR utilizing fresh-frozen tissue has a 10- to 50- fold greater sensitivity for detection of chimeric gene fusions in soft tissue sarcomas than RT-PCR using paraffin-embedded tissue. ${ }^{41}$ Similar results to those achieved in frozen tissues can be attained from archival material by the addition of a nested RT-PCR step, ${ }^{17}$ but this is time consuming and labor intensive. In addition, the high sensitivity of this method may increase false-positive results. In contrast to cytogenetics and RT-PCR, FISH represents a cost- and time-efficient method, which can be undertaken utilizing paraffin-embedded, formalin-fixed tissue. Our laboratory has found that tissue microarrays assembled from formalin-fixed tissue are a useful tool for evaluating immunohistochemical stains and for performing FISH. Tissue microarrays allow for evaluation of large numbers of cases, are cost-effective in that they require less probe and reagents, are less time consuming for the technologist, conserve tissue, and yield reproducible results. ${ }^{42}$

Our study demonstrates that dual-color, breakapart, interphase FISH detects EWS gene rearrangement in a substantial proportion of clear cell sarcomas, with excellent specificity, a finding consistent with previous studies. ${ }^{30,34}$ The relative lack of sensitivity of this probe may be explained by variant translocations, or an alternative molecular pathogenesis in the FISH-negative tumors; further studies of these cases utilizing alternative methods are indicated. In this series, three clear cell sarcomas were found to be negative for the EWS rearrangement by FISH. As two of these cases were scored from the tissue microarray, conventional whole histologic sections from these cases were subjected to repeat analysis by FISH and were negative for EWS rearrangement with repeat testing. The third FISH-negative case had correlative negative RT-PCR for the EWS-ATF1 fusion transcript. Given the sensitivity and specificity of the probe in this series, we believe that these are true fusion-negative cases.

The most important finding in this study is that $E W S$ FISH is negative in malignant melanoma and therefore highly specific for clear cell sarcoma in this differential diagnosis. FISH can be performed on uncultured cells, including those from frozen tissue. However, in cases where fresh tissue is unavailable, FISH evaluation of formalin-fixed, paraffin-embedded tissue appears to be an important ancillary diagnostic test, in that it represents an alternative to RT-PCR for making the distinction between clear cell sarcoma and its most important mimic, malignant melanoma.

\section{Duality of interest}

Authors have no duality of interest.

\section{References}

1 Enzinger FM. Clear cell sarcoma of tendons and aponeuroses: an analysis of 21 cases. Cancer 1965; 18:1163-1174.

2 Chung EB, Enzinger FM. Malignant melanoma of soft parts. A reassessment of clear cell sarcoma. Am J Surg Pathol 1983;7:405-413.

3 Pavlidis NA, Fisher C, Wiltshaw E. Clear-cell sarcoma of tendons and aponeuroses: a clinicopathologic study. Presentation of six additional cases with review of the literature. Cancer 1984;54:1412-1417.

4 Sara AS, Evans HL, Benjamin RS. Malignant melanoma of soft parts (clear cell sarcoma). A study of 17 cases, with emphasis on prognostic factors. Cancer 1990;65:367-374.

5 Deenik W, Mooi WJ, Rutgers EJ, et al. Clear cell sarcoma (malignant melanoma) of soft parts: a clinicopathologic study of 30 cases. Cancer 1999;86: 969-975.

6 Kindblom LG, Lodding P, Angervall L. Clear-cell sarcoma of tendons and aponeuroses. An immunohistochemical and electron microscopic analysis indicating neural crest origin. Virchows Arch A Pathol Anat Histopathol 1983;401:109-128.

7 Swanson PE, Wick MR. Clear cell sarcoma: an immunohistochemical analysis of six cases and comparison with other epithelioid neoplasms of soft tissue. Arch Pathol Lab Med 1989;113:55-60.

8 Granter SR, Weilbaecher KN, Quigley C, et al. Clear cell sarcoma shows immunoreactivity for microphthalmia transcription factor: further evidence for melanocytic differentiation. Mod Pathol 2001;14:6-9.

9 Chang KL, Folpe AL. Diagnostic utility of microphthalmia transcription factor in malignant melanoma and other tumors. Adv Anat Pathol 2001;8:273-275.

10 Moritake H, Sugimoto T, Asada Y, et al. Newly established clear cell sarcoma (malignant melanoma of soft parts) cell line expressing melanoma-associated 
melan-A antigen and overexpressing C-MYC oncogene. Cancer Genet Cytogenet 2002;135:48-56.

11 Bridge JA, Sreekantaiah C, Neff JR, et al. Cytogenetic findings in clear cell sarcoma of tendons and aponeuroses. Malignant melanoma of soft parts. Cancer Genet Cytogenet 1991;52:101-106.

12 Stenman G, Kindblom LG, Angervall L. Reciprocal translocation $\mathrm{t}(12 ; 22)(\mathrm{q} 13 ; \mathrm{q} 13)$ in clear-cell sarcoma of tendons and aponeuroses. Genes Chromosomes Cancer 1992;4:122-127.

13 Reeves BR, Fletcher CD, Gusterson BA. Translocation $\mathrm{t}(12 ; 22)(\mathrm{q} 13 ; \mathrm{q} 13)$ is a nonrandom rearrangement in clear cell sarcoma. Cancer Genet Cytogenet 1992;64: 101-103.

14 Travis JA, Bridge JA. Significance of both numerical and structural chromosomal abnormalities in clear cell sarcoma. Cancer Genet Cytogenet 1992;64:104-106.

15 Pellin A, Monteagudo C, Lopez-Gines C, et al. New type of chimeric fusion product between the EWS and ATFI genes in clear cell sarcoma (malignant melanoma of soft parts). Genes Chromosomes Cancer 1998;23: 358-360.

16 Langezaal SM, Graadt van Roggen JF, Cleton-Jansen AM, et al. Malignant melanoma is genetically distinct from clear cell sarcoma of tendons and aponeurosis (malignant melanoma of soft parts). Br J Cancer 2001; 84:535-538.

17 Antonescu CR, Tschernyavsky SJ, Woodruff JM, et al. Molecular diagnosis of clear cell sarcoma: detection of EWS-ATF1 and MITF-M transcripts and histopathological and ultrastructural analysis of 12 cases. J Mol Diagn 2002;4:44-52.

18 Panagopoulos I, Mertens F, Debiec-Rychter M, et al. Molecular genetic characterization of the EWS/ATF1 fusion gene in clear cell sarcoma of tendons and aponeuroses. Int J Cancer 2002;99:560-567.

19 Graadt van Roggen JF, Mooi WJ, Hogendoorn PC. Clear cell sarcoma of tendons and aponeuroses (malignant melanoma of soft parts) and cutaneous melanoma: exploring the histogenetic relationship between these two clinicopathological entities. J Pathol 1998; 186:3-7.

20 Barth A, Wanek LA, Morton DL. Prognostic factors in 1521 melanoma patients with distant metastases. [see comment]. J Am Coll Surg 1995;181:193-201.

21 Lucas DR, Nascimento AG, Sim FH. Clear cell sarcoma of soft tissues. Mayo Clinic experience with 35 cases. Am J Surg Pathol 1992;16:1197-1204.

22 Liu WW, Skacel M, Hicks DG, et al. Initial experience with a novel fluorescence in situ hybridization (FISH) assay for detection of Ewing sarcoma (EWS) gene (22q12) translocations in paraffin-embedded tissue (abstract). Mod Pathol 2004;17:1511A

23 Weiss SW, Goldblum JR. Enzinger and Weiss's Soft Tissue Tumors, 4th edn. Mosby: St Louis, MO, 2001, p 1242.

24 Mooi WJ, Deenik W, Peterse JL, et al. Keratin immunoreactivity in melanoma of soft parts (clear cell sarcoma). Histopathology 1995;27:61-65.

25 Takahira T, Oda Y, Tamiya S, et al. Alterations of the p16INK4a/p14ARF pathway in clear cell sarcoma. Cancer Sci 2004;95:651-655.

26 Segal NH, Pavlidis P, Noble WS, et al. Classification of clear-cell sarcoma as a subtype of melanoma by genomic profiling. J Clin Oncol 2003;21:1775-1781.

27 Sandberg AA, Bridge JA. Updates on the cytogenetics and molecular genetics of bone and soft tissue tumors: clear cell sarcoma (malignant melanoma of soft parts). Cancer Genet Cytogenet 2001;130:1-7.

28 Bastian BC, Xiong J, Frieden IJ, et al. Genetic changes in neoplasms arising in congenital melanocytic nevi: differences between nodular proliferations and melanomas. Am J Pathol 2002;161:1163-1169.

29 Maize JC, McCalmont TH, Carlson JA, et al. Genomic analysis of blue nevi and related dermal melanocytic proliferations. Am J Surg Pathol 2005;29:1214-1220.

30 Speleman F, Delattre O, Peter M, et al. Malignant melanoma of the soft parts (clear-cell sarcoma): confirmation of EWS and ATF-1 gene fusion caused by a t(12;22) translocation. Mod Pathol 1997;10:496-499.

31 Bridge JA, Borek DA, Neff JR, et al. Chromosomal abnormalities in clear cell sarcoma. Implications for histogenesis. Am J Clin Pathol 1990;93:26-31.

32 Hiraga H, Nojima T, Abe S, et al. Establishment of a new continuous clear cell sarcoma cell line. Morphological and cytogenetic characterization and detection of chimaeric EWS/ATF-1 transcripts. Virchows Arch 1997;431:45-51.

33 Mrozek K, Karakousis CP, Perez-Mesa C, et al. Translocation $\mathrm{t}(12 ; 22)(\mathrm{q} 13 ; \mathrm{q} 12.2-12.3)$ in a clear cell sarcoma of tendons and aponeuroses. Genes Chromosomes Cancer 1993;6:249-252.

34 Zucman J, Delattre O, Desmaze C, et al. EWS and ATF1 gene fusion induced by $\mathrm{t}(12 ; 22)$ translocation in malignant melanoma of soft parts. Nat Genet 1993;4: 341-345.

35 Sciot R, Speleman F. Clear cell sarcoma of soft tissue. In: Fletcher CD, Unni KK, Mertens F (eds). Pathology and Genetics: Tumors of Soft Tissue and Bone. IARC Press: Lyon, 2002, pp 211-212.

36 Fujimura Y, Ohno T, Siddique $\mathrm{H}$, et al. The EWS-ATF1 gene involved in malignant melanoma of soft parts with $\mathrm{t}(12 ; 22)$ chromosome translocation, encodes a constitutive transcriptional activator. Oncogene 1996; 12:159-167.

37 Fujimura Y, Siddique H, Lee L, et al. EWS-ATF-1 chimeric protein in soft tissue clear cell sarcoma associates with CREB-binding protein and interferes with p53-mediated trans-activation function. Oncogene 2001;20:6653-6659.

38 Panagopoulos I, Mertens F, Isaksson M, et al. Absence of mutations of the BRAF gene in malignant melanoma of soft parts (clear cell sarcoma of tendons and aponeuroses). Cancer Genet Cytogenet 2005;156:74-76.

39 Schaefer KL, Brachwitz K, Wai DH, et al. Expression profiling of $\mathrm{t}(12 ; 22)$ positive clear cell sarcoma of soft tissue cell lines reveals characteristic up-regulation of potential new marker genes including ERBB3. Cancer Res 2004;64:3395-3405.

40 Stegnaier S, Leuschner I, Aakcha-Rudel E, et al. Identification of various exon combinations of the ews/fli1 translocation: an optimized RT-PCR method for paraffin embedded tissue-a report by the CWSstudy group. Klin Padiatr 2004;216:315-322.

41 Jin L, Majerus J, Oliveira A, et al. Detection of fusion gene transcripts in fresh-frozen and formalin-fixed paraffin-embedded tissue sections of soft-tissue sarcomas after laser capture microdissection and rt-PCR. Diag Mol Pathol 2003;12:224-230.

42 Skacel M, Skilton B, Pettay JD, et al. Tissue microarrays: a powerful tool for high-throughput analysis of clinical specimens: a review of the method with validation data. Appl Immunohistochem Mol Morphol 2002;10:1-6. 